\title{
Electronic Structure and Optical Properties of Co and Fe doped $\mathrm{ZnO}$
}

\author{
Chunping $\mathrm{LI}^{1, \mathrm{a} *}{ }^{*} \mathrm{Ge} \mathrm{GAO}{ }^{2}$, and Xin $\mathrm{CHEN}^{2}$ \\ ${ }^{1}$ Aviation University of Air force, Changchun 130022, China \\ ${ }^{2}$ Changchun University of Science and Technology, Changchun 130022, China \\ a1cp0431@126.com
}

\begin{abstract}
First-principle ultrasoft pseudo potential approach of the plane wave based on density functional theory has been used for studying the electronic characterization and optical properties of $\mathrm{ZnO}$ and $\mathrm{Fe}$, Co doped $\mathrm{ZnO}$. The results show that the doping impurities change the lattice parameters a little, but bring more changes in the electronic structures. The band gaps are broadened by doping. And the Fermi level accesses to the conduction band which will lead the system to show the character of metallic properties. The dielectric function and absorption peaks are identified and the changes compared to pure $\mathrm{ZnO}$ are analyzed in detail. Keywords: First-principle, $\mathrm{ZnO}$, Electronic structure, Optical properties
\end{abstract}

\section{Introduction}

Diluted magnetic semiconductors have become a supernova in semiconductor industry with their unique features lately. They are promising candidates for the next generation of new functional devices, and are imagined to be applied in High-density non-volatile memory unit, spintronic devices, magnetic sensors, optical isolation device, quantum computers, etc [1-5].

For better applications of preparing various devices, the optical properties of $\mathrm{ZnO}$-based dilute magnetic semiconductors have been the focus of study. Cheng X.W.et al. has studied the magnetic mechanism and emission characters of Co-doped $\mathrm{ZnO}$ polycrystalline with sol-gel method, and has found that the ferromagnetism was originated in $\mathrm{Zn}_{\mathrm{i}}, \mathrm{Co}$ exists in the way of substituting $\mathrm{Zn}$ in $\mathrm{ZnO}$ that changed the band gap [6]. S. Sain, et al. have investigated both the microstructural and optical characterizations of nanocrystalline $\mathrm{Zn}_{1-\mathrm{x}} \mathrm{Mn}_{\mathrm{x}} \mathrm{O}$ that prepared by mechanical alloying, and analyzed the course of the decreased of bad gap. More of the reports are based on experimental research and there exists many restrictions [7]. The theoretical study can exclude the interference of external conditions and can guarantee the comparable conditions, but the relevant theoretical research is still limited. In this article, we use first principles based on density functional method to study the electronic and optical properties of $\mathrm{Fe}$, Co doped $\mathrm{ZnO}$ and the comparable results are also analyzed here.

* Corresponding author: 1cp0431@126.com 


\section{Computational Details}

All calculations were implemented in the module of CASTEP from Materials Studio 6.1, which is based on the plane wave pseudopotential Density Function Theory. Ideal ZnO possesses a hexagonal wurtzite structure and belongs to P63mc space group, $C_{6 v}^{4}$ symmetry system. The values of cell parameters are as follow: $\mathrm{a}=\mathrm{b}=0.3249 \mathrm{~nm}, \mathrm{c}=0.5206 \mathrm{~nm}$, $\mathrm{c} / \mathrm{a}=1.602$, which is less than the ideal 1.633. $\alpha=\beta=90^{\circ}, \gamma=120^{\circ}$. All configurations contains 16 atoms, they are $8 \mathrm{Zn}$ and $8 \mathrm{O}$ atoms, equals to a system of $2 \times 2 \times 1$ supercell with periodic boundary conditions. The $\mathrm{Zn}$ atom was substituted by $\mathrm{Fe}$ or $\mathrm{Co}$ when doping, which formed a constitution of $12.5 \%$ single-doped system. The GGA of Perdew Burke and Ernzerh of (PBE) is used for handling the electronic exchange-correlation function [4]. The valence states we chosen for atomic pseudopotential calculations are: $\mathrm{O} 2 \mathrm{~s}^{2} 2 \mathrm{p}^{4}, \mathrm{Zn} 3 \mathrm{~d}^{10} 4 \mathrm{~s}^{2}$, Fe $3 d^{6} 4 s^{2}, \quad \operatorname{Co} 3 d^{7} 4 s^{2}$. The $4 \times 4 \times 5$ Monkhorst-Pack k-points grid was adopted for integration in the first Brillouin zone. The cutoff energy was $340 \mathrm{eV}$. We chose the Broyden-Fletcher-Goldfarb-Shanno (BFGS) for a faster geometry optimization to obtain a lowest energy structure. The total energy is converged to less than $1 \times 10^{-6} \mathrm{eV}$.

\section{Results and discussion}

First is about the Lattice parameters. Table 1 presents the lattice parameter of the geometry-optimized $\mathrm{ZnO}$ and its doping system, which showed us that different doping element present minimal impact on the changing rate of the lattice parameters a and c. And the ratio between them floats up and down slightly. With the incorporation of new elements, a tiny change we can see in its volume. Maybe it was caused by the different ionic radii of $\mathrm{Fe}$ and Co compared with $\mathrm{Zn}$.

Table 1. Lattice Parameters Of Zn1-Xmxo ( $\mathrm{M}=\mathrm{Fe}, \mathrm{Co}, \mathrm{X}=0,0.125)$

\begin{tabular}{|l|l|l|l|l|}
\hline & $\mathrm{a}$ & $\mathrm{c}$ & $\mathrm{c} / \mathrm{a}$ & $\mathrm{V}$ \\
\hline $\mathrm{ZnO}$ & 3.288 & 5.307 & 1.61 & 198.863 \\
\hline $\mathrm{ZnFeO}$ & 3.296 & 5.274 & 1.60 & 198.285 \\
\hline $\mathrm{ZnCoO}$ & 3.295 & 5.267 & 1.60 & 198.180 \\
\hline
\end{tabular}

Second is about the Band structure. Band structure can bring us some information about the electronic structure of the crystal. For better comparison among the pure system and doped system, we had calculated band structure of pure $\mathrm{ZnO}$ before other analyses, as shown in Fig.1 (a). Fig.1 (b) and (c) present the calculated energy band structures of

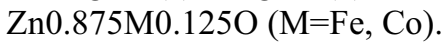



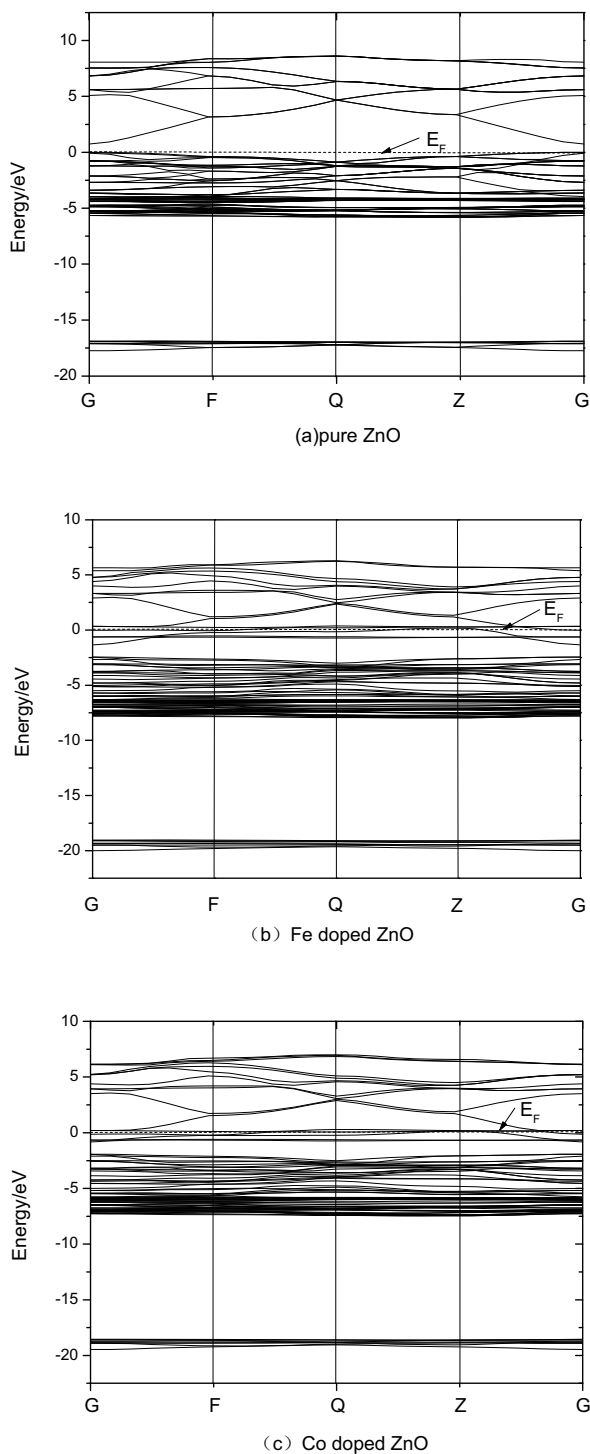

Fig 1 Band structures: (a) Pure $\mathrm{ZnO}$; (b) Fe doped $\mathrm{ZnO}$; (c) Co doped $\mathrm{ZnO}$

The calculated band gap is $0.735 \mathrm{eV}$ for pure $\mathrm{ZnO}$. This result somewhat agrees well with the reported theoretical result by Gao X.Q. et al. [1]. However, it is obviously beyond the experimental value $3.37 \mathrm{eV}$. This underestimation of band gap may coursed by the defective of GGA itself [2]. And more details for pure $\mathrm{ZnO}$ are that the overestimated contribution from $\mathrm{Zn} 3 \mathrm{~d}$ state has enhanced the interaction with $\mathrm{O} 2 \mathrm{p}$ state. Thus, it increased the valence band, followed with the decreased band gap. Nevertheless, it will not affect the results of electronic structure and other properties analyzation. From the figure we can see that unadulterated $\mathrm{ZnO}$ crystal has a direct band gap. The minimum of conduction band and the maximum valence band are all located at the k-point $(G)$ in Brillouin zone. Fig.1 (b), (c) had brought us a information that there was a movement of Fermi level to the conduction band in both two condition above compared with pure $\mathrm{ZnO}$, and showed a transformation to metallic. 
The band gaps are $1.107 \mathrm{eV}$ and $1.04 \mathrm{eV}$ for $\mathrm{Zn}_{0.875} \mathrm{Fe}_{0.125} \mathrm{O}$ and $\mathrm{Zn}_{0.875} \mathrm{Co}_{0.125} \mathrm{O}$, respectively. The broadening band gaps may cause by the high carrier concentration which brought the Fermi level to the conduction band, it was called Burstein-moss [3].

Third is about Density of states. To illustrate contributions of each electron orbital to energy band, we've exhibited the total density of states (DOS) of pure $\mathrm{ZnO}$ together with the partial density of states (PDOS) of zinc and oxygen for further analysis as shown in Fig.2. We can clear see that Fermi level set in 0 point of energy is plotted by dashed line. And between $-17.6 \mathrm{eV}$ to $16.4 \mathrm{eV}$ exists a insufficiently distinctive narrow band interacts with the valence band, which is mainly formed by $\mathrm{O}(2 \mathrm{~s})$ state of electrons. This part is far with the others in valence band and nature of the material has little impacts on the overall situation. The two zones of valence band:-3 0 eV and $-6 \sim 3 \mathrm{eV}$ are derived from $\mathrm{O}(2 \mathrm{p})$ state and $\mathrm{Zn}$ (3d) state separately. Energy band locals at the bottom of the conduction band that mainly composed of $\mathrm{Zn}(4 \mathrm{~s})$ state accompany with a significant transition process between $\mathrm{Zn}(4 \mathrm{~s})$ and $\mathrm{O}(2 \mathrm{p})$ state.

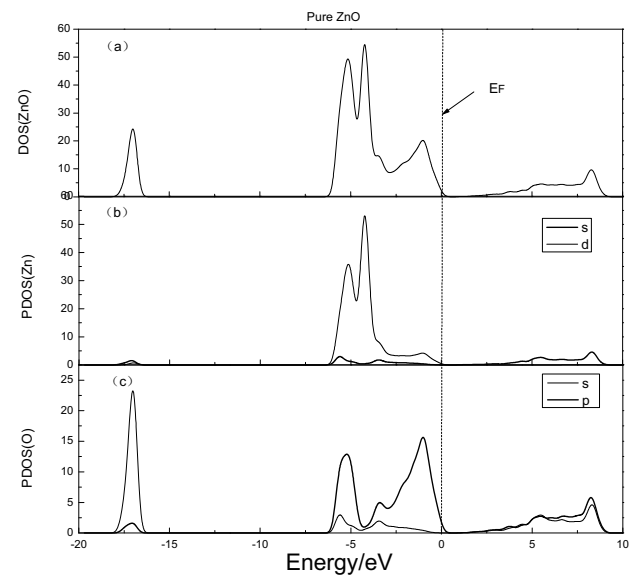

Fig. 2 DOS and PDOS of pure $\mathrm{ZnO}$ 

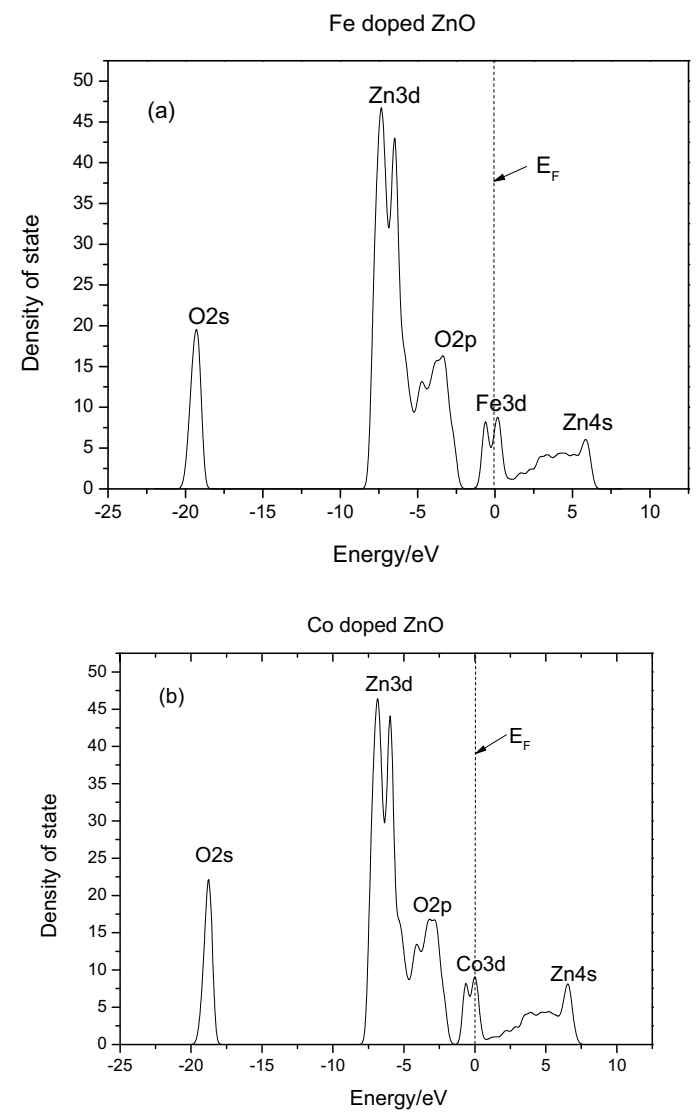

Fig. 3 DOS of (a) Fe doped $\mathrm{ZnO}$, (b) Co doped $\mathrm{ZnO}$

Fig.3 gives the density of states of $\mathrm{Fe}$ and $\mathrm{Co}$ doped $\mathrm{ZnO}$. Together with Fig.1, we've found that Fermi level access into the conduction band and the system shows the character of metallic properties, when the Fe or Co replaced the position of $\mathrm{Zn}$ locations. We can also find that the localized $3 \mathrm{~d}$ electron from substituting dopant ions for $\mathrm{Zn}$ just locals at the Fermi level. And the conduction band edge moves by the reason of exchanging-interaction of electronic energy band between s-p and $p-d 3 d$ state from the dopant atoms occurs orbital hybridization with $\mathrm{O} 2 \mathrm{p}$ state. Orbit superposition becomes more serious and the rejection is reduced then, which will lead to the shift of optical absorption edge to the lower energy.

The fourth is about the optical properties. To achieve better investigation of semiconductor materials, optical properties play a very important role. It connects well with the micro structure of physical process and the solid microelectronic structures. Hence, we can get more details of the materials. And the optical dielectric function is just like a bridge well characterized the solids band structure and varieties of spectral information. We can use the complex dielectric constant $\varepsilon(\omega)=\varepsilon_{1}(\omega)+\varepsilon_{2}(\omega)$ to describe the macroscopic optical response function of solid in the linear response, in which $\varepsilon_{1}=n^{2}-k^{2}$ is the real part that we obtain it according to the Kramers-Kronig dispersion relations [1], and $\varepsilon_{2}=2 n k$ is the imaginary part. Then, we can calculate the absorption coefficient. The main results are as follows: 


$$
\begin{gathered}
\varepsilon_{2}=\frac{4 \pi^{2}}{m^{2} \omega^{2}} \sum_{V, C} \int_{B Z} d^{3} k \frac{2}{2 \pi}\left|e \cdot M_{C V}(k)\right|^{2} \times \delta\left[E_{C}(K)-E_{V}(K)-\eta \omega\right] \\
\varepsilon_{1}=1+\frac{8 \pi^{2} e^{2}}{m^{2}} \sum_{V, C} d^{3} k \frac{2}{2 \pi} \times \frac{\left|e \cdot M_{C V}(k)\right|^{2}}{\left[E_{C}(K)-E_{V}(K)\right]} \times \frac{\eta^{3}}{\left[E_{C}(K)-E_{V}(K)-\eta^{2} \omega^{2}\right]} \\
I(\omega)=\sqrt{2} \omega\left[\sqrt{\varepsilon_{1}(\omega)^{2}-\varepsilon_{2}(\omega)^{2}}-\varepsilon_{1}(\omega)\right]^{1 / 2}
\end{gathered}
$$
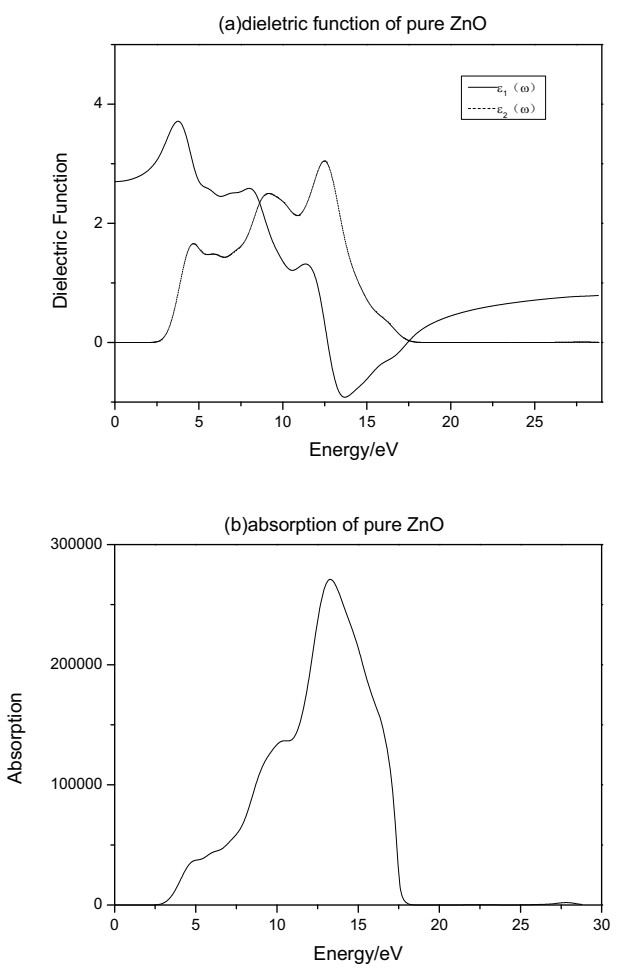

Fig.4 (a) Dielectric function of $\mathrm{ZnO}$; (b) Absorption of pure $\mathrm{ZnO}$

Fig.4 (a) shows us the dielectric function diagram of pure $\mathrm{ZnO}$. Solid line represents the real part of the dielectric function and dot line exhibits the imaginary part of dielectric function. The scissors of $2.635 \mathrm{eV}$ was used in calculation. As we can see in the figure that there are three absorption peak of the imaginary part as follows: $4.66 \mathrm{eV}, 9.10 \mathrm{eV}$ and 12.47 eV. Combined with Fig.3, we can figure out that the peak of $4.66 \mathrm{eV}$ mainly coursed by the electronic transition between the two orbital energy levels, which are $\mathrm{O}(2 \mathrm{p})$ state from the top of the valence band and $\mathrm{Zn}(4 \mathrm{~s})$ state from the bottom of the conduction band. Peak located at $9.10 \mathrm{eV}$ was formed by the transition between $\mathrm{Zn} \mathrm{(3d)} \mathrm{and} \mathrm{O} \mathrm{(2p).} \mathrm{The} \mathrm{strongest}$ peak of $12.47 \mathrm{eV}$ was from the transition between $\mathrm{Zn}(3 \mathrm{~d})$ and $\mathrm{O}(2 \mathrm{~s})$. We can get the static dielectric constant $\varepsilon_{0}=2.701$ from the solid line. The minimum photon energy is located at $13.7 \mathrm{eV}$, on the left of which shows a downward trend. There was a significantly enhance of electronic inter-band transitions. From Fig.4(b) we can see that there also exists three mainly peaks at $4.7 \mathrm{eV}, 10.2 \mathrm{eV}$ and $13.2 \mathrm{eV}$, separately. The peaks have a tiny deviation 
corresponding to peaks in the imaginary part of dielectric function. This may be coursed by ignorance of the relaxation effects during electron transition process.

Comparing with that in pure $\mathrm{ZnO}$, Fig.5 (a) exhibits the imaginary part of dielectric function of $\mathrm{Fe}$, Co doped $\mathrm{ZnO}$. We can clearly see in Fig.5 (a) that the peak at about $3 \mathrm{eV}$ has enhanced because of the $\mathrm{Fe}$ and Co impurities. The impurities provides the $4 \mathrm{~s}$ electrons and increases with the transition of O2p. And the peak shows a slight movement trends to lower energy direction. The peak may cause by the electron transition between $\mathrm{O} 2 \mathrm{p}$ state and $\mathrm{Zn} 4 \mathrm{~s}$ state binding with the band structure information of each system. Fig.5 (b) shows the absorption changes when doped with Fe and Co. Corresponding to Fig. 5(a), we can also see a trend of movement of the peak to lower energy region in both two doping conditions. The absorption edge shows a red shift. The new peak appears at about $3.53 \mathrm{eV}$ and $3.77 \mathrm{eV}$ in the absorption spectrum may be caused by the $\mathrm{d}$-d transition of the impurity ions.
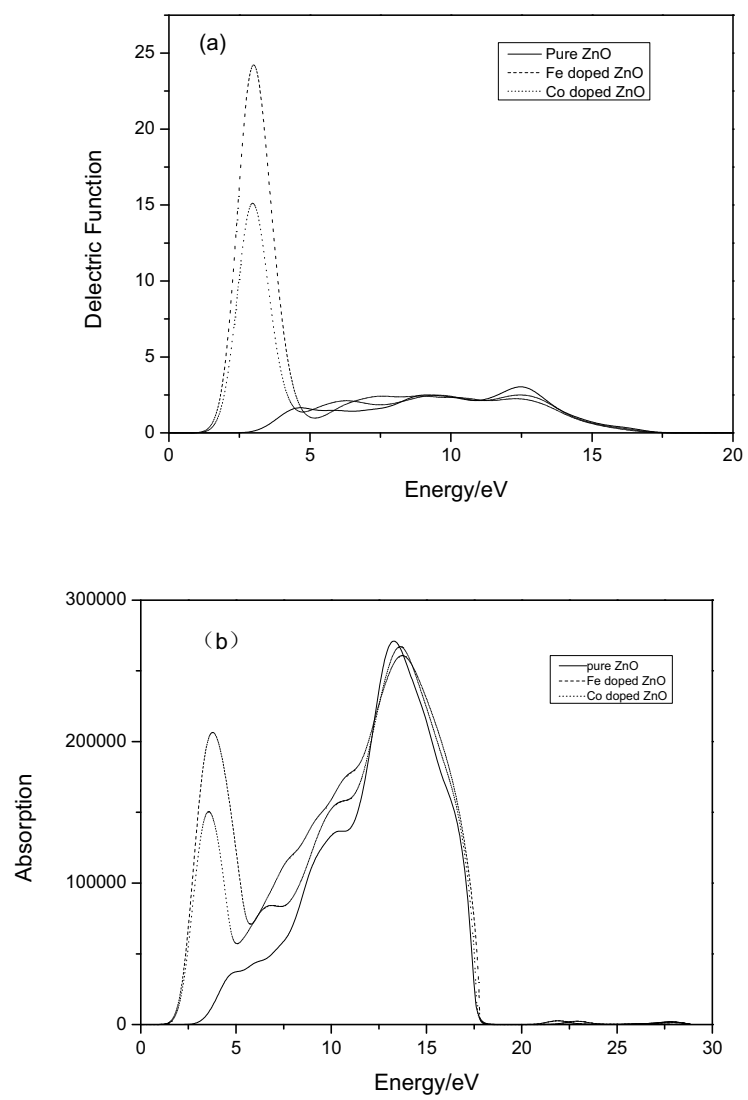

Fig. 5 (a) Imaginary part of dielectric function; (b) Absorption

\section{Conclusion}

Ultrasoft pseudo potential approach of the plane wave and GGA based on DFT has been used in the study. We have both investigated the electronic structure and the optical properties of pure $\mathrm{ZnO}$ and $\mathrm{Fe}, \mathrm{Co}$ doped $\mathrm{ZnO}$. The results shows that the broadening of the band gaps of doped $\mathrm{ZnO}$ may cause by the Burstein-moss effect. And the Fermi level 
accesses into the conduction band which will lead the system to show the character of metallic properties. The dielectric function and absorption peaks are identified by considering the electronic transition, and some peaks are enhanced because of the $\mathrm{Fe}$ and $\mathrm{Co}$ impurities. Orbit superposition results in the shift of optical absorption edge to the lower energy.

\section{Acknowledgements}

This work was financially supported by the National Natural Science Foundation of China under Grant No. 51272285 and the Twelfth Five-Year Science and Technology Research Project of Jilin Provincial Department of Education under Grant No. 2013-520.

\section{References}

1. Gao Xiaoqi, Guo Zhiyou,et al., The electronic structure and optical properties of Al-N codoped ZnO, Chinese Journal of Luminescence, 31, 4 (2010) 509-514.

2. P. Palacios, K. Sánchez, P. Wahnón, Ab-initio valence band spectra of A1, In doped ZnO, Thin Solid Films, 517 (2009) 2448-2451.

3. E. Burstein. Anomalous optical absorption limit in InSb [J] Phys.Rev, 93(1954) 632-633.

4. Duan Man-Yi, Xu Ming, Zhou Hai-Ping et al., First-principles study on the electronic structure and optical properties of $\mathrm{ZnO}$ doped with transition metal and N. ACTA PHYSICA SINICA, 56, 9 (2007) 5359-5365.

5. Y. Xu, B. Yao, Y.F. Li, Z.H. Ding, J.C. Li, et al., Chemical states of gold doped in ZnO films and its effect on electrical and optical properties, Journal of Alloys and Compounds, 585 (2014) 479-484.

6. Cheng Xing-Wang, Li Xiang, Gao Yuan-Ling, Yu Zhou, Long Xue, Liu Ying, Synthesis and magnetic, optical properties of $\mathrm{Co}$ doped $\mathrm{ZnO}$ room-temperature ferromagnetic semiconductor, Acta Phys. Sin., 58, 3 (2009) 2018-2022.

7. Chunping Li, Li Zhang, Changjie Liu, Ge Gao, Exciton localization and stimulated emission of ZnO nanorods, Key Engineering Materials, 538 (2013) 161-164. 